\title{
Auger Recombination in GaAs from First Principles
}

\author{
Daniel Steiauf, ${ }^{* \dagger}$ Emmanouil Kioupakis, ${ }^{*},+\dagger$ and Chris G. Van de Walle*,† \\ ${ }^{\dagger}$ Materials Department, University of California, Santa Barbara, California 93106-5050, United States \\ ${ }^{\ddagger}$ Department of Materials Science and Engineering, University of Michigan, Ann Arbor, Michigan 48109, United States
}

ABSTRACT: Auger recombination is a significant loss mechanism in many optoelectronic devices. We use first-principles methods based on density functional theory to study the relative importance of direct and indirect phonon-assisted Auger recombination in GaAs and related alloys. Energy and momentum of the recombining electron-hole pair can be transferred to an Auger electron (eeh process) or an Auger hole (hhe process). For eeh processes, the direct process is negligibly small compared to the phonon-assisted indirect process in GaAs, while in hhe processes the direct and phonon-assisted processes contribute almost equally. The hole processes are about 5 times stronger than the electron processes. In alloys with lower band gaps, the eeh processes become stronger, and below a band gap of
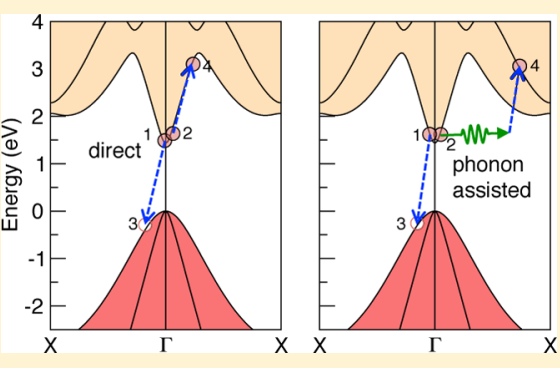
$0.8 \mathrm{eV}$ they are as relevant as the hole processes. Our results highlight the importance of indirect processes, even at low band gaps.

KEYWORDS: Auger recombination, phonons, efficiency droop, density functional theory, electron-phonon coupling, gallium arsenide

allium arsenide and its alloys are widely used in optoelectronic devices such as light-emitting and laser diodes. ${ }^{1,2}$ The internal quantum efficiency of these devices, defined as the fraction of electron-hole pairs that recombine by emitting a photon, is reduced by various nonradiative recombination processes. Auger recombination ${ }^{3}$ is such a nonradiative process that affects the efficiency of devices operating at high power. ${ }^{4,5}$ Auger recombination has been identified as a probable cause of the efficiency-droop and greengap problems of nitride light-emitting diodes. ${ }^{6-9}$ The investigation of the microscopic origins of Auger recombination in semiconductors can thus yield valuable information about carrier recombination in optoelectronic devices at high power.

In the Auger process, an electron and a hole recombine nonradiatively by transferring their energy and momentum to a third carrier (Figure 1a). For the electron-electron-hole (eeh) Auger process this third carrier is another electron, while for hole-hole-electron (hhe) Auger the third carrier is a hole. As there are three carriers involved in the recombination, the rate is proportional to $n^{2} p$ for eeh and to $n p^{2}$ for hhe, where $n$ is the electron and $p$ is the hole concentration. Under deviceoperating conditions the electrically or optically injected electron and hole concentrations are usually equal to each other and exceed those introduced by doping. In this case the rates are given by $C_{n} n^{3}$ (eeh) and $C_{p} n^{3}$ (hhe), where $C_{n}$ and $C_{p}$ are the corresponding Auger coefficients. This dependence indicates that Auger recombination becomes increasingly important at high carrier densities.

Auger recombination in GaAs can occur in a direct fashion (Figure 1a), or it can be mediated by lattice vibrations (Figure 1b). The additional momentum provided by emission or absorption of a phonon makes significantly more final states accessible for Auger transitions and enables Auger transitions that would otherwise be forbidden due to energy and
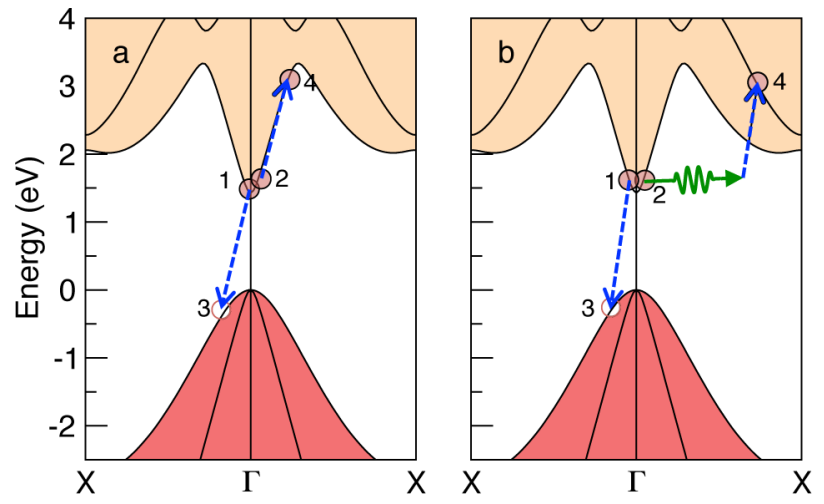

Figure 1. Schematic illustration of (a) direct and (b) indirect electron-electron-hole Auger recombination superimposed on the calculated band structure of GaAs. (a) In the direct case electron 1 and hole 3 recombine while transferring their energy to electron 2 and exciting it to conduction-band state 4. (b) In the indirect case, phonon-assisted Auger recombination is enabled by the additional momentum provided by lattice vibrations.

momentum conservation. First-principles calculations have shown that in $\mathrm{GaN}$ the phonon-assisted Auger recombination rate is several orders of magnitude higher than the direct recombination rates. ${ }^{7}$ An interesting open question is whether Auger recombination in GaAs is dominated by direct or phonon-assisted microscopic processes.

Auger recombination in GaAs has been the subject of several experimental $^{10-12}$ and theoretical ${ }^{13-18}$ studies, but its microscopic mechanism remains unclear. Experiments ${ }^{11,12}$ have yielded values for Auger coefficients ranging from $7 \times 10^{-30}$

Received: April 11, 2014

Published: July 21, 2014 
to $1.6 \times 10^{-29} \mathrm{~cm}^{-3}$ (Table 1). However, experimental studies have difficulty distinguishing among the various microscopic

Table 1. Theoretical Values and Experimental Measurements for the Direct and Phonon-Assisted Electron-ElectronHole (eeh) and Hole-Hole-Electron (hhe) Auger Recombination Coefficients in GaAs at Room Temperature

\begin{tabular}{llr}
\multicolumn{1}{c}{ method } & \multicolumn{1}{c}{ Auger type } & $C\left(\mathrm{~cm}^{6} \mathrm{~s}^{-1}\right)$ \\
this work & direct eeh & $<10^{-33}$ \\
& phonon eeh & $1.1 \times 10^{-31}$ \\
& direct hhe & $2.2 \times 10^{-31}$ \\
& phonon hhe & $3.1 \times 10^{-31}$ \\
& direct eeh & $5 \times 10^{-34}$ \\
calculated $^{a}$ & direct hhe & $8.13 \times 10^{-31}$ \\
& total eeh & $\leq 1.6 \times 10^{-29}$ \\
experiment $^{b}$ & total eeh+hhe & $(7 \pm 4) \times 10^{-30}$ \\
experiment $^{c}$ & direct eeh & 0 \\
model theory $^{d}$ & phonon eeh & $4.72 \times 10^{-30}$ \\
& direct hhe & $4.64 \times 10^{-31}$ \\
& phonon hhe & $6.4 \times 10^{-31}$ \\
& direct + phonon eeh & $1.7 \times 10^{-31}$ \\
model theory $^{e}$ & direct + phonon hhe & $2.4 \times 10^{-30}$
\end{tabular}

${ }^{a}$ Ref 18; for hhe the value at a carrier concentration of $10^{18} \mathrm{~cm}^{-3}$ is given. ${ }^{b}$ Ref $12 .{ }^{c} \operatorname{Ref} 11 .{ }^{d} \operatorname{Ref} 16 .{ }^{e} \operatorname{Ref} 15$.

Auger processes. Theoretical investigations, on the other hand, have produced conflicting results about the relative importance of direct versus phonon-assisted Auger recombination. Early theoretical studies ${ }^{13-16}$ noted that direct eeh Auger in GaAs is weak. However, these early studies used model parameters to describe the electronic and vibrational properties of GaAs and considered only a subset of the vibrational modes and diagrammatic terms involved. Phonon-assisted Auger recombination rates in GaAs have not been calculated from first principles to date.

In this work, we investigate direct and phonon-assisted Auger recombination in GaAs using first-principles calculations based on density functional theory (DFT), following the methodology of ref 7. The single-particle energies and wave functions are calculated with $\mathrm{DFT}^{19,20}$ in the local density approximation (LDA), ${ }^{21,22}$ using the plane-wave pseudopotential method ${ }^{23,24}$ as implemented in the Quantum Espresso code. ${ }^{25}$ A constant scissors shift is applied to the energies of the unoccupied states to correct for the band gap underestimation of LDA.

The direct Auger recombination rate is determined from time-dependent perturbation theory. ${ }^{17,26}$ The screened-Coulomb-interaction matrix elements are obtained based on a model dielectric function ${ }^{27}$ and the experimental dielectric constant of GaAs. Local field effects are neglected in the model function but were concluded not to be important for the calculation of Auger rates in GaAs. ${ }^{18}$ The Fermi occupation numbers for the electrons and holes are calculated for a free carrier density of $10^{18} \mathrm{~cm}^{-3}$ and a temperature of $T=300 \mathrm{~K}$. Spin-orbit coupling is neglected, as it was in previous calculations. ${ }^{17,18}$ The split-off band at $350 \mathrm{meV}$ below the valence-band maximum is not occupied with free holes, so it is not available as an initial hole state. Also, because of energy conservation, the split-off band is not available as a final hole state close to the zone center. A $80 \times 80 \times 80 \mathrm{k}$-point mesh was used to sample the first Brillouin zone for the calculation of the direct Auger recombination rate.
The phonon-assisted recombination rate is calculated with second-order time-dependent perturbation theory. To keep the numerical complexity of the calculations within the limits of available CPU time, the initial-state wave functions of the recombining electron and hole are approximated by those at the band extrema at the $\Gamma$ point. This approximation is also justified from finding all initial states of the direct calculation close to the zone center when partial thermal occupancies are correctly taken into account. The vibrational properties and electron-phonon matrix elements are obtained from density functional perturbation theory. ${ }^{28}$ Phonons and electronic states are calculated on a $56 \times 56 \times 56$ k-point mesh in the first Brillouin zone. A coarser k-point mesh than in the direct case is sufficient because the indirect Auger recombination rate shows fewer features as a function of energy. The phonon Bose occupation numbers are also calculated at $T=300 \mathrm{~K}$.

Our calculated values for the direct and phonon-assisted Auger coefficients of GaAs at the experimental band gap of 1.43 $\mathrm{eV}$ for both eeh and hhe processes are listed in Table 1 and compared to experiment and to previous theoretical approaches. Our results show that the contribution of the direct eeh process to the total Auger rate is negligibly small, as the corresponding Auger coefficient $\left(<10^{-33} \mathrm{~cm}^{6} \mathrm{~s}^{-1}\right)$ is at least 2 orders of magnitude smaller that phonon-assisted eeh Auger $\left(1.1 \times 10^{-31} \mathrm{~cm}^{6} \mathrm{~s}^{-1}\right)$. This agrees with the very small direct eeh Auger coefficient found in ref $18\left(5 \times 10^{-34} \mathrm{~cm}^{6} \mathrm{~s}^{-1}\right)$. For the hhe case the direct (purely Coulomb) Auger coefficient $(2.2$ $\left.\times 10^{-31} \mathrm{~cm}^{6} \mathrm{~s}^{-1}\right)$ is larger than for the eeh case $\left(<10^{-33} \mathrm{~cm}^{6}\right.$ $\mathrm{s}^{-1}$ ), and the indirect phonon-assisted hhe coefficient is of the same order of magnitude as the direct hhe coefficient $(3.1 \times$ $\left.10^{-31} \mathrm{~cm}^{6} \mathrm{~s}^{-1}\right)$. The indirect phonon-assisted eeh coefficient $\left(1.1 \times 10^{-31} \mathrm{~cm}^{6} \mathrm{~s}^{-1}\right)$ is much larger than the direct eeh, but less than half as large as the hhe indirect phonon-assisted. Our values agree with the experimental values within an order of magnitude.

Figure 2 shows the relative contributions of phonons to the eeh and hhe Auger recombination rates as a function of the

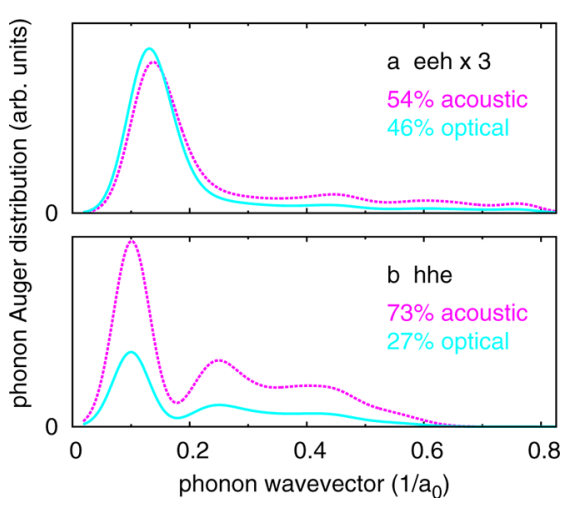

Figure 2. Distribution of contributions of acoustic (dashed magenta) and optical (solid cyan) phonons with different wavevectors to the phonon-assisted Auger recombination rate for (a) eeh processes and (b) he processes in GaAs.

phonon wavevector. The rates from acoustic or optical phonons are given by the areas of the respective curves in Figure 2. For both eeh and hhe processes the distributions show a peak at short wavevectors. The first peak corresponds to a phonon wavelength of $8 a_{0}$ for eeh and $10 a_{0}$ for hhe recombination, where $a_{0}$ is the Bohr radius. The rate coefficients can also be divided into contributions from acoustic 
and optical phonons. For eeh indirect phonon-assisted Auger recombination, acoustic and optical phonons contribute almost equally, while for hhe processes the acoustic contribution is more than twice as strong as the optical. The distribution with respect to wavevector does not significantly depend on the mode character.

Our results allow us to estimate the direct and indirect phonon-assisted Auger coefficients of GaAs-based alloys. This is done by adjusting the band gap with the scissors shift over a range of energies around the experimental gap of GaAs (1.43 $\mathrm{eV}$ ) to approximate the alloy band structure while keeping the Coulomb and electron-phonon matrix elements fixed. This is a good approximation for compositions close to GaAs, but might become worse when the band gap and alloy composition changes considerably from pure GaAs. (Aside from the effect of the band gap on the energy and momentum conservation, there is an additional scattering mechanism introduced when translational symmetry is broken by the structural disorder in an alloy. ${ }^{7}$ This additional indirect contribution from the alloy disorder scattering is not considered here.) The results are shown in Figure 3. For band gaps smaller than GaAs (as would

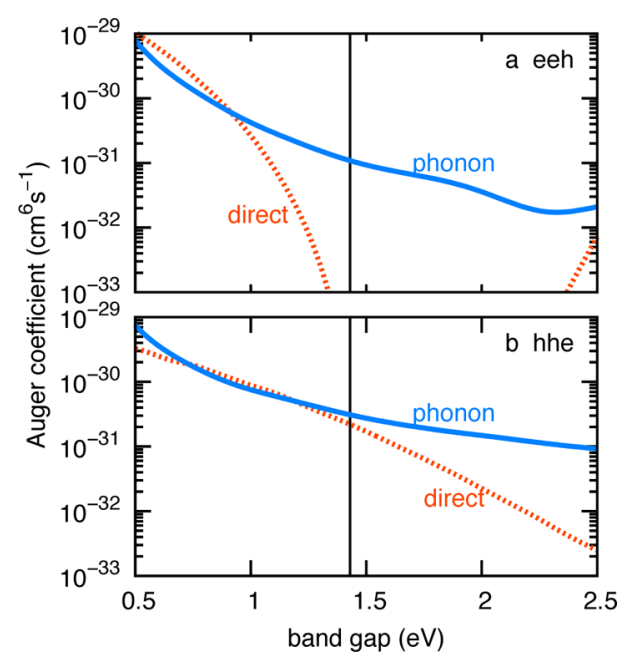

Figure 3. Auger recombination rate coefficient vs band gap for direct (dotted, orange) and phonon-assisted (solid, blue) Auger recombination: (a) eeh and (b) hhe recombination processes. The vertical line is at the experimental band gap of GaAs.

be achieved in alloys of GaAs with InAs or GaSb), the direct Auger coefficient increases with decreasing band gap. Figure 3 shows that the phonon-assisted rates follow the same trend when the gap is reduced. For the hhe process, the phononassisted process results in a rate that remains comparable to the direct rate for smaller band gaps. For the eeh process, it is only at gaps below $1 \mathrm{eV}$ that the direct process becomes larger than the phonon-assisted indirect process, and even then by only a small amount. The largest ratio between direct to phononassisted Auger recombination that we found is only 1.8 for eeh processes and only 1.2 for hhe processes. At the experimental band gap, hhe processes are about 5 times stronger than eeh, while for lower band gaps this ratio decreases. Below $0.8 \mathrm{eV}$ the eeh rate coefficients are larger than the hhe. For band gaps larger than $\mathrm{GaAs}$ (as would occur in alloys of $\mathrm{GaAs}$ with $\mathrm{GaP}$ or AlAs), the coefficient of the direct process is significantly smaller than that of the phonon-assisted process, for both eeh and he recombination.
In conclusion, we use first-principles calculations to evaluate the purely Coulomb (direct) and the phonon-assisted (indirect) Auger recombination rate in GaAs and related alloys. For pure GaAs, direct eeh Auger processes are negligible. The direct hhe Auger rate is more than 2 orders of magnitude larger than the eeh rate and is comparable to the phonon-assisted hhe rate. Direct Auger becomes increasingly important as a function of decreasing band gap, for both eeh and hhe processes. The phonon-assisted contributions also increase with decreasing band gap, and only considering the direct process is not sufficient even in GaAs-based low-band-gap alloys. Phononassisted Auger recombination clearly dominates over direct Auger in wider-band-gap GaAs alloys. The analysis of our results shows that it is important to include all phonon modes for phonon-assisted indirect recombination. Restricting the modeling of phonon scattering to a specific mode, e.g., LO (longitudinal optical), would leave important contributions unaccounted for. Our results identify the dominant microscopic Auger recombination mechanism in GaAs and related materials that affects the high-power performance of optoelectronic devices.

\section{AUTHOR INFORMATION}

\section{Corresponding Authors}

*E-mail: steiauf@engineering.ucsb.edu.

*E-mail: kioup@umich.edu.

*E-mail: vandewalle@mrl.ucsb.edu.

\section{Notes}

The authors declare no competing financial interest.

\section{ACKNOWLEDGMENTS}

This work was supported as part of the Center for Energy Efficient Materials, an Energy Frontier Research Center funded by the U.S. Department of Energy, Office of Science, Basic Energy Sciences, under Award Number DE-SC0001009. It used resources of the National Energy Research Scientific Computing Center, which is supported by the Office of Science of the U.S. Department of Energy under Contract No. DE-AC0205CH11231. Additional computational resources were provided by the Center for Scientific Computing at the CNSI and MRL (an NSF MRSEC, DMR-1121053) (NSF CNS0960316).

\section{REFERENCES}

(1) Schubert, E. F. Light-Emitting Diodes; Cambridge University Press, 2006.

(2) Coldren, L. A.; Corzine, S. W. Diode Lasers and Photonic Integrated Circuits; Wiley, 1995.

(3) Ridley, B. K. Quantum Processes in Semiconductors; Clarendon Press, Oxford, 1982.

(4) Horikoshi, Y. In Lightwave Communications Technology Semiconductor Injection Lasers, II Light-Emitting Diodes; Tsang, W., Ed.; Semiconductors and Semimetals; Elsevier, 1985; Vol. 22, Part C; pp 93-151.

(5) Fehse, R.; Tomic, S.; Adams, A.; Sweeney, S.; O’Reilly, E.; Andreev, A.; Riechert, H. A Quantitative Study of Radiative, Auger, and Defect Related Recombination Processes in 1.3- $\mu \mathrm{m}$ GaInNAsBased Quantum-Well Lasers. IEEE J. Sel. Top. Quantum Electron. 2002, $8,801-810$

(6) Shen, Y. C.; Mueller, G. O.; Watanabe, S.; Gardner, N. F.; Munkholm, A.; Krames, M. R. Auger Recombination in InGaN Measured by Photoluminescence. Appl. Phys. Lett. 2007, 91, 141101. 
(7) Kioupakis, E.; Rinke, P.; Delaney, K. T.; Van de Walle, C. G. Indirect Auger Recombination as a Cause of Efficiency Droop in Nitride Light-Emitting Diodes. Appl. Phys. Lett. 2011, 98, 161107.

(8) Kioupakis, E.; Yan, Q.; Van de Walle, C. G. Interplay of Polarization Fields and Auger Recombination in the Efficiency Droop of Nitride Light-Emitting Diodes. Appl. Phys. Lett. 2012, 101, 231107.

(9) Iveland, J.; Martinelli, L.; Peretti, J.; Speck, J. S.; Weisbuch, C. Direct Measurement of Auger Electrons Emitted from a Semiconductor Light-Emitting Diode under Electrical Injection: Identification of the Dominant Mechanism for Efficiency Droop. Phys. Rev. Lett. 2013, 110, 177406.

(10) Marín, E.; Riech, I.; Díaz, P.; Alvarado-Gil, J. J.; Baquero, R.; Mendoza-Alvarez, J. G.; Vargas, H.; Cruz-Orea, A.; Vargas, M. Photoacoustic Determination of Non-Radiative Carrier Lifetimes. J. Appl. Phys. 1998, 83, 2604-2609.

(11) Strauss, U.; Rühle, W. W.; Köhler, K. Auger Recombination in Intrinsic GaAs. Appl. Phys. Lett. 1993, 62, 55-57.

(12) Lush, G. B.; MacMillan, H. F.; Keyes, B. M.; Levi, D. H.; Melloch, M. R.; Ahrenkiel, R. K.; Lundstrom, M. S. A Study of Minority Carrier Lifetime versus Doping Concentration in n-Type GaAs Grown by Metalorganic Chemical Vapor Deposition. J. Appl. Phys. 1992, 72, 1436-1442.

(13) Lochmann, W. Phonon-Assisted Auger Recombination in Semiconductors. Phys. Status Solidi A 1977, 40, 285-292.

(14) Lochmann, W. Scattering Mechanisms in Phonon-Assisted Auger Recombination. Phys. Status Solidi A 1977, 42, 181-185.

(15) Takeshima, M. Simple Method of Calculating Phonon-Assisted Auger Recombination Rate in Direct-Gap Semiconductors. Jpn. J. Appl. Phys. 1983, 22, 491-498.

(16) Haug, A. Auger Recombination in Direct-Gap Semiconductors: Band-Structure Effects. J. Phys. C: Solid State Phys. 1983, 16, 41594172.

(17) Picozzi, S.; Asahi, R.; Geller, C. B.; Freeman, A. J. Accurate FirstPrinciples Detailed-Balance Determination of Auger Recombination and Impact Ionization Rates in Semiconductors. Phys. Rev. Lett. 2002, 89, 197601.

(18) Govoni, M.; Marri, I.; Ossicini, S. Auger Recombination in Si and GaAs Semiconductors: Ab Initio Results. Phys. Rev. B 2011, 84, 075215.

(19) Hohenberg, P.; Kohn, W. Inhomogeneous Electron Gas. Phys. Rev. 1964, 136, B864-B871.

(20) Kohn, W.; Sham, L. J. Self-Consistent Equations Including Exchange and Correlation Effects. Phys. Rev. 1965, 140, A1133A1138.

(21) Ceperley, D. M.; Alder, B. J. Ground State of the Electron Gas by a Stochastic Method. Phys. Rev. Lett. 1980, 45, 566-569.

(22) Perdew, J. P.; Zunger, A. Self-Interaction Correction to DensityFunctional Approximations for Many-Electron Systems. Phys. Rev. B 1981, 23, 5048-5079.

(23) Ihm, J.; Zunger, A.; Cohen, M. L. Momentum-Space Formalism for the Total Energy of Solids. J. Phys. C: Solid State Phys. 1979, 12, $4409-4422$.

(24) Troullier, N.; Martins, J. L. Efficient Pseudopotentials for PlaneWave Calculations. Phys. Rev. B 1991, 43, 1993-2006.

(25) Giannozzi, P.; Baroni, S.; Bonini, N.; Calandra, M.; Car, R.; Cavazzoni, C.; Ceresoli, D.; Chiarotti, G. L.; Cococcioni, M.; Dabo, I.; Corso, A. D.; de Gironcoli, S.; Fabris, S.; Fratesi, G.; Gebauer, R.; et al. QUANTUM ESPRESSO: A Modular and Open-Source Software Project for Quantum Simulations of Materials. J. Phys.: Condens. Matter 2009, 21, 395502.

(26) Laks, D. B.; Neumark, G. F.; Pantelides, S. T. Accurate Interband-Auger-Recombination Rates in Silicon. Phys. Rev. B 1990, 42, 5176-5185.

(27) Cappellini, G.; Del Sole, R.; Reining, L.; Bechstedt, F. Model Dielectric Function for Semiconductors. Phys. Rev. B 1993, 47, 98929895.

(28) Baroni, S.; de Gironcoli, S.; Dal Corso, A.; Giannozzi, P. Phonons and Related Crystal Properties from Density-Functional Perturbation Theory. Rev. Mod. Phys. 2001, 73, 515-562. 